\title{
ГНСС-ИЗМЕРЕНИЯ В ПОЛЯРНЫХ ШИРОТАХ: СРАВНЕНИЕ ГЕОМЕТРИИ РАСПОЛОЖЕНИЯ СПУТНИКОВ НА ПУНКТАХ ФАГС БАРЕНЦБУРГ И НОВОСИБИРСК
}

\section{Елена Геннадвевна Гиенко}

Сибирский государственный университет геосистем и технологий, 630108, Россия, г. Новосибирск, ул. Плахотного, 10, кандидат технических наук, доцент, доцент кафедры космической и физической геодезии, тел. (913)376-52-24, e-mail: elenagienko@yandex.ru

\section{Анастасия Сергеевна Саливончик}

Сибирский государственный университет геосистем и технологий, 630108, Россия, г. Новосибирск, ул. Плахотного, 10, магистрант кафедры космической и физической геодезии, тел. (913)338-48-76, e-mail: nsalivonchik@mail.ru

Представлены результаты расчетов количества спутников GPS и ГЛОНАCC, а также геометрических факторов, снижающих точность местоопределения, для пунктов ФАГС Баренцбург и Новосибирск. Показано, что на пункте Баренцбруг ( $78^{0}$ с.ш.) наблюдается большее количество спутников, и в северном направлении - на большей высоте, чем в Новосибирске $\left(55^{0}\right.$ с.ш.). На пункте Баренцбург величина геометрического фактора PDOP для суммарного созвездия спутников GPS и ГЛОНАСС не превышает значения 4 в течение суток в разные сезоны, что является хорошим показателем, при этом для созвездия исключительно спутников ГЛОНАСС в течение суток могут быть кратковременные увеличения геометрического фактора до 6 - 10 единиц. Этот факт следует учитывать при планировании ГНСС-измерений, навигации по спутникам ГЛОНАСС и реализации метода точного точечного позиционирования в северных широтах, а также при обновлении или расширении орбитальной группировки ГЛОНАСС.

Ключевые слова: ГНСС-измерения, геометрический фактор ГНСС, фундаментальная астрономо-геодезическая сеть ФАГС, созвездие спутников, GPS, ГЛОНАСС

\section{GNSS MEASUREMENTS AT POLAR LATITUDES: COMPARISON OF THE SATELLITE CONSTELLATION GEOMETRY AT BARENTSBURG AND NOVOSIBIRSK FAGN STATIONS}

\section{Elena G. Gienko}

Siberian State University of Geosystems and Technologies, 10, Plakhotnogo St., Novosibirsk, 630108, Russia, Ph. D., Associate Professor, Department of Space and Physical Geodesy, e-mail: elenagienko@yandex.ru

\section{Anastasiya S. Salyvonchik}

Siberian State University of Geosystems and Technologies, 10, Plakhotnogo St., Novosibirsk, 630108, Russia, Master Student, phone (913) 338-48-76, e-mail: nsalivonchik@mail.ru

The results of calculations of GPS and GLONASS satellites number, as well as geometric factors that reduce the accuracy of positioning, for the Barentsburg and Novosibirsk FAGN stations are presented. It is shown that there are more satellites at the Barentsbrug station $(78 \mathrm{~N})$, and in the north direction - at a higher elevation than in Novosibirsk (55N). At the Barentsburg station, the geometric PDOP factor for the total constellation of GPS and GLONASS does not exceed 4 during 
the day in different seasons, which is a good indicator, while for the exclusively GLONASS constellation, there may be short-term increases in the geometric factor up to 6-10 units during the day. This fact should be taken into account when planning GNSS measurements, navigating GLONASS satellites and implementing the method of precise point positioning in Northern latitudes, as well as when updating or expanding the GLONASS.

Keywords: GNSS measurements, GNSS geometric factor, fundamental astronomical and FAGN geodetic network, constellation of satellites, GPS, GLONASS

\section{Введение}

- Как известно, взаимное расположение спутников ГНСС (геометрический фактор [4]) может по-разному влиять на качество определения координат заданной точки. Величина геометрического фактора напрямую связана с числом обусловленности системы уравнений наблюдений. Теория и алгоритм расчета геометрических факторов снижения точности (Dilution of Precision, DOP) изложены в монографии [12] и учебном пособии [1].

- В Инструкции [3], опубликованной в 2003 г., необходимым этапом технологии ГНСС-измерений для создания съемочного обоснования, съемки ситуации и рельефа являлось предварительное прогнозирование спутникового созвездия (планирование). Здесь же было рекомендовано ограничение на величину геометрического фактора - не более 7.

- В технических требованиях к пунктам высокоточной геодезической сети [5] установлено ограничение геометрического фактора PDOP не более 4 при маске по высоте $10^{0}$ и продолжительности сеанса не менее 3 суток с наблюдением созвездия спутников GPS и ГЛОНАСС; технические требования к пунктам ФАГС [6] ограничивают геометрический фактор значением 3.

- В настоящее время, при полном развертывании глобальных навигационных спутниковых систем, количество наблюдаемых спутников в любой точке земного шара достаточно для круглосуточного навигационного решения с геометрическим фактором, как правило, не превышающим значения 3. Поэтому в современных нормативных документах по применению ГНСС для решения задач геодезии и навигации [8] уже не регламентируются предельные значения геометрических факторов, а само прогнозирование спутникового созвездия выполняется в редких случаях - при наличии препятствий, закрывающих радиогоризонт.

- Однако в северных районах, в Заполярье - на значительной части России расположение спутников ГНСС может быть не столь удачным, и актуальной задачей является расчет геометрических факторов на данных территориях, с последующими рекомендациями по выполнению ГНСС-измерений. Результаты расчета значений РDОР для созвездия ГЛОНАСС позволят дать заключение о полноте и достаточности отечественной орбитальной группировки для навигационного обеспечения северных районов. Кроме того, полученные результаты могут быть полезны для развития метода точного точечного позиционирования (Precise Point Positioning, PРP), [2, 7, 13] в северных широтах. 


\section{Эксперименты}

Для решения поставленной задачи были выбраны два пункта фундаментальной астрономо-геодезической сети (ФАГС) - заполярный Баренцбург и, для сравнения, Новосибирск. Информация о пунктах ФАГС представлена на сайте Центра геодезии, картографии и инфраструктуры геопространственных данных (ЦГКиИПД) [10], а также доступна на сайте RGS-center [9].

Пункт ФАГС Баренцбург (BARE), расположенный на архипелаге Шпицберген (Норвегия, демилитаризованная зона), рис.1, является самым северным пунктом фундаментальной астрономо-геодезической сети $\left(78^{0}\right.$ с.ш.), где с октября 2018 года выполняются постоянные ГНСС-измерения по наблюдениям спутников GPS и ГЛОНАСС.

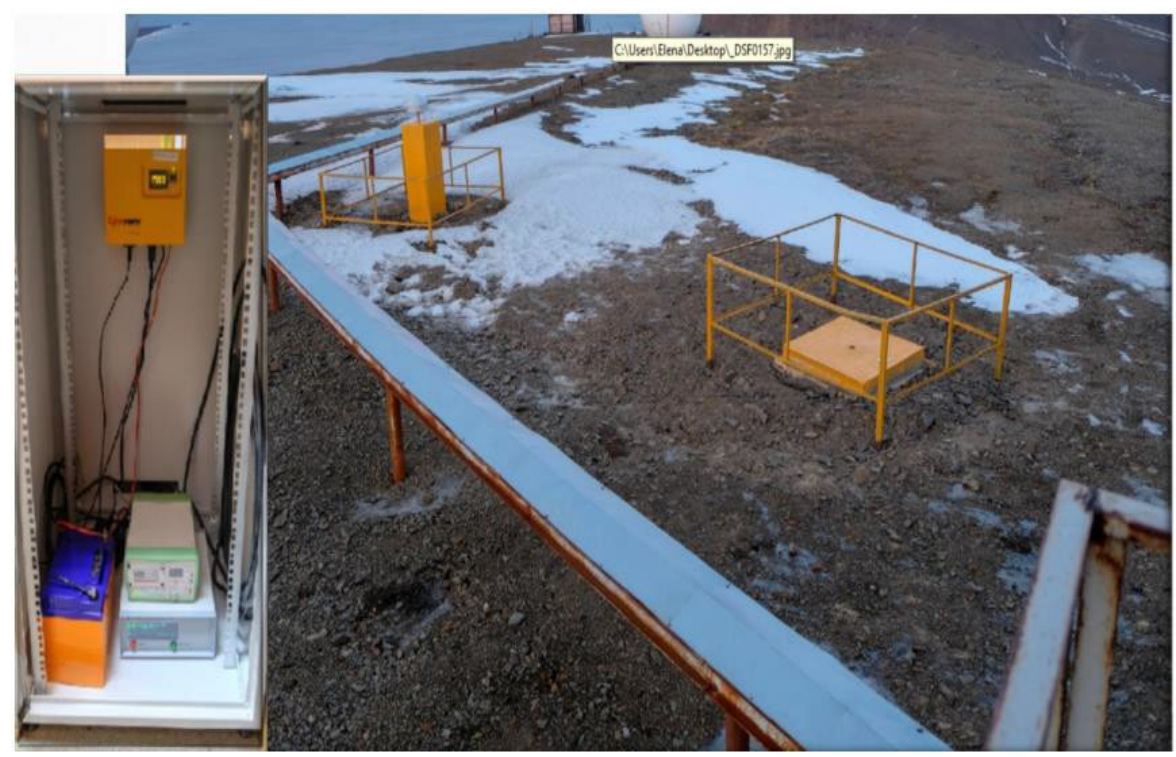

Рис. 1. Пункт ФАГС Баренцбург [11]

- Пункт ФАГC NSK1 находится в городе Новосибирск (55 с.ш.). Основные сведения о пунктах NSK1 и BARE приведены в табл. 1.

Таблица 1

Сведения о пунктах BARE и NSK1. Геоцентрические координаты XYZ приведены в системе ГСК-2011 на эпоху 2011.01.01

\begin{tabular}{|c|c|c|c|c|c|c|}
\hline Код & \multicolumn{3}{|c|}{ Координаты } & \multicolumn{2}{c|}{ Скорости/данные о ГНСС-измерениях } \\
\hline \multirow{5}{*}{ BARE } & $\mathrm{X}, \mathrm{m}$ & $\mathrm{Y}, \mathrm{m}$ & $\mathrm{Z}, \mathrm{m}$ & $\mathrm{Vx}, \mathrm{м} /$ год & Vy, м/год & $\mathrm{Vz}, \mathrm{m} /$ год \\
\cline { 2 - 7 } & 1282847,960 & 325537,340 & 6218685,230 & - & - & - \\
\cline { 2 - 7 } & $\mathrm{B}$ & $\mathrm{L}$ & $\mathrm{H}$ & Антенна & Приемник & $\begin{array}{c}\text { Стандарт } \\
\text { частоты }\end{array}$ \\
\cline { 2 - 7 } & $78^{\circ} 03^{\prime} 48^{\prime \prime}$ & $14^{\circ} 14^{\prime} 20^{\prime \prime}$ & $290 \mathrm{~m}$ & LEIAR20 LEIM & $\begin{array}{c}\text { LEICA } \\
\text { GR50 }\end{array}$ & внутренний \\
\hline
\end{tabular}


Окончание табл. 1

\begin{tabular}{|c|c|c|c|c|c|c|}
\hline Код & \multicolumn{3}{|c|}{ Координаты } & \multicolumn{2}{c|}{ Скорости/данные о ГНСС-измерениях } \\
\hline \multirow{5}{*}{ NSK1 } & $\mathrm{X}, \mathrm{м}$ & $\mathrm{Y}, \mathrm{m}$ & $\mathrm{Z}, \mathrm{m}$ & $\mathrm{Vx,} \mathrm{м/год}$ & Vу, м/год & $\mathrm{Vz}$, м/год \\
\cline { 2 - 7 } & 447670,304 & 3638117,385 & 5202281,564 & $-0,0260$ & 0,0039 & 0,0008 \\
\cline { 2 - 7 } & $\mathrm{B}$ & $\mathrm{L}$ & $\mathrm{H}$ & Антенна & Приемник & $\begin{array}{c}\text { Стандарт } \\
\text { частоты }\end{array}$ \\
\cline { 2 - 7 } & $55^{\circ} 00^{\prime} 44^{\prime \prime}$ & $82^{\circ} 59^{\prime} 6^{\prime \prime}$ & $141 \mathrm{~m}$ & $\begin{array}{c}\text { JAV_RINGANT_G3T } \\
\text { JAVC }\end{array}$ & $\begin{array}{c}\text { TRE_G3T } \\
\text { H_5 }\end{array}$ & внутренний \\
\hline
\end{tabular}

Для расчетов использовалось открытое программное обеспечение Trimble GNSS Planning [14], где для пунктов BARE и NSK1 было определено количество наблюдаемых спутников и вычислены геометрические факторы снижения точности положения в пространстве (Position Dilution of Precision, PDOP), по высоте (Vertical DOP, VDOP), в плане (Horizontal DOP, HDOP) на двух суточных интервалах (02.01.2020 и 01.07.2020). Расчеты были выполнены для каждого из созвездий спутников GPS, ГЛОНАСС, а также для общего созвездия (GPS и ГЛОНАСС). Маска по высоте была установлена $10^{\circ}$.

\section{Обсуждение}

Результаты расчетов в программе Trimble GNSS Planning представляются в виде графиков количества спутников, геометрических факторов и карт неба с треками спутников. По данной информации были выписаны максимальное и минимальное значение числа наблюдаемых спутников (табл. 2) и геометрических факторов (табл. 4) на двух пунктах ФАГС.

Таблий 2

Результаты расчетов количества спутников в ПО Trimble GNSS Planning

\begin{tabular}{|c|c|c|c|c|c|c|}
\hline Даты & \multicolumn{9}{|c|}{02.01 .2020} & \multicolumn{3}{|c|}{01.07 .2019} \\
\hline ГНСС & \multirow{2}{*}{ GPS } & ГЛОНАСС & GPS+ГЛОНАСС & GPS & ГЛОНАСС & GPS+ГЛОНАСС \\
\hline \multicolumn{7}{|c|}{ ВARE } \\
\hline $\min$ & 8 & 6 & 15 & 7 & 7 & 15 \\
\hline $\max$ & 12 & 10 & 21 & 13 & 10 & 21 \\
\hline \multicolumn{7}{|c|}{ NSK1 } \\
\hline $\min$ & 6 & 5 & 12 & 6 & 6 & 13 \\
\hline $\max$ & 11 & 10 & 20 & 12 & 9 & 20 \\
\hline
\end{tabular}

Как видно из табл. 2, на обоих пунктах наблюдается достаточное количество спутников, как GPS, так и ГЛОНАСC (в сумме от 15 до 21 на пункте BARE и от 13 до 20 на пункте NSK1), что связано с полным развертыванием 
этих ГНСС. Вопреки ожиданиям, на северном пункте BARE наблюдается больше спутников ГНСС, чем на расположенном в средних широтах пункте NSK1.

По картам неба (рис. 2) видно, что на пункте BARE спутники ГНСC наблюдаются на высоте более $30^{\circ}$ практически во всех направлениях, в отличие от пункта NSK1, где в северном направлении спутники не видны, и количество спутников на 1-2 меньше. Визуально (рис. 2) хорошая геометрия расположения спутников над пунктом BARE обеспечивается созвездием ГЛОНАCС, благодаря большему наклону орбиты этих спутников. С другой стороны, спутников ГЛОНАСС наблюдается меньше, чем GPS, на обоих пунктах. Совместное использование спутников GPS и ГЛОНАСС дает хорошие результаты.

Максимальные высоты спутников в северном и южном направлении можно рассчитать на основании наклона орбиты и широты пункта. В табл. 3 приведены результаты расчетов высот спутников над горизонтом в верхней и нижней кульминациях для пунктов NSK1 и BARE.

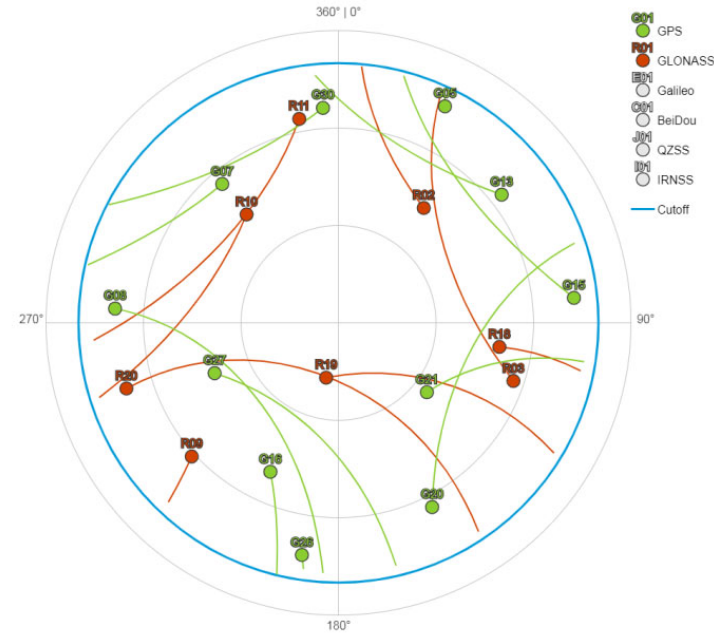

BARE

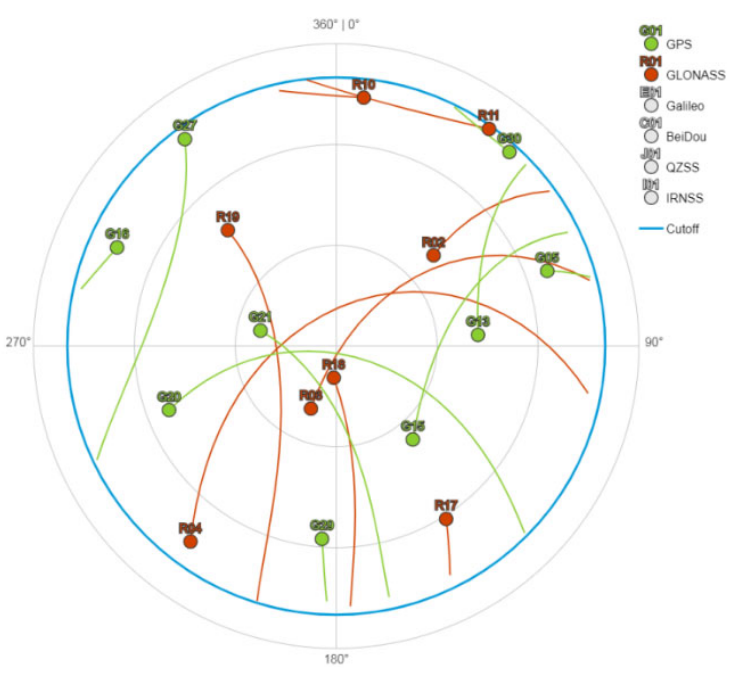

NSK1

Рис. 2. Карта неба на пунктах BARE и NSK1 (02.01.2020), Trimble GNSS Planning

Таблица 3

Высота спутников в верхней и нижней кульминации (ВК и НК)

\begin{tabular}{|c|c|c|c|c|}
\hline \multirow{2}{*}{ Высота, } & \multicolumn{2}{|c|}{ ВАRE } & \multicolumn{2}{c|}{ NSK1 } \\
\cline { 2 - 5 } & GPS & ГЛОНАСС & GPS & ГЛОНАСС \\
\hline ВК & 67 & 76,8 & 90 (в зените) & 80,2 (с северной стороны) \\
\hline НК & 43 & 52,8 & 20 & 29,8 \\
\hline
\end{tabular}

Результаты расчетов геометрических факторов PDOP, VDOP, HDOP приведены в табл. 4. 
Таблица 4

Результаты расчетов геометрических факторов в ПО Trimble GNSS Planning

\begin{tabular}{|c|c|c|c|c|c|c|}
\hline Даты & \multicolumn{7}{|c|}{02.01 .2020} & \multicolumn{3}{c|}{01.07 .2019} \\
\hline ГНСС & GPS & ГЛОНАСС & GPS+ГЛОНАСС & GPS & ГЛОНАСС & GРS+ГЛОНАСС \\
\hline \multicolumn{7}{|c|}{ BАRE } \\
\hline PDOP(min) & 1,76 & 1,73 & 1,38 & 1,66 & 1,72 & 1,41 \\
\hline PDOP(max) & 4,92 & 10,10 & 3,26 & 4,30 & 3,92 & 2,72 \\
\hline VDOP(max) & 4,21 & 8,05 & 2,33 & 3,63 & 3,06 & 1,93 \\
\hline VDOP(min) & 1,39 & 1,30 & 0,97 & 1,31 & 1,30 & 0,99 \\
\hline HDOP(max) & 1,22 & 2,18 & 0,72 & 1,17 & 1,31 & 0,76 \\
\hline HDOP(min) & 0,70 & 0,78 & 0,54 & 0,68 & 0,80 & 0,53 \\
\hline \multicolumn{7}{|c|}{ NSK1 } \\
\hline PDOP(min) & 1,45 & 1,55 & 1,26 & 1,54 & 1,68 & 1,36 \\
\hline PDOP(max) & 4,32 & 6,00 & 2,43 & 4,47 & 3,29 & 2,28 \\
\hline VDOP(max) & 3,2 & 2,88 & 1,82 & 3,13 & 2,33 & 1,5 \\
\hline VDOP(min) & 1,14 & 1,19 & 0,83 & 1,08 & 1,17 & 0,88 \\
\hline HDOP(max) & 2,28 & 1,85 & 1,23 & 2,16 & 1,57 & 0,94 \\
\hline HDOP(min) & 0,78 & 0,84 & 0,59 & 0,79 & 0,85 & 0,58 \\
\hline
\end{tabular}

Как видно из таблицы 4, геометрический фактор PDOP для спутников GPS и ГЛОНАСС составляет в среднем величину 2,5, и не превышает значения 4, как для пункта BARE, так и для пункта NSK1. Однако при расчетах для спутников ГЛОНАСС на пункте BARE на дату 02.01.2020 фактор PDOP имеет максимальное значение 10,1 в течение 0,5 часа, а также есть три промежутка времени, когда его величина достигает 6,7 и 5,5 (рис. 3).Подобное увеличение значения геометрического фактора следует учитывать при планировании ГНССизмерений спутников ГЛОНАСС в северных широтах.

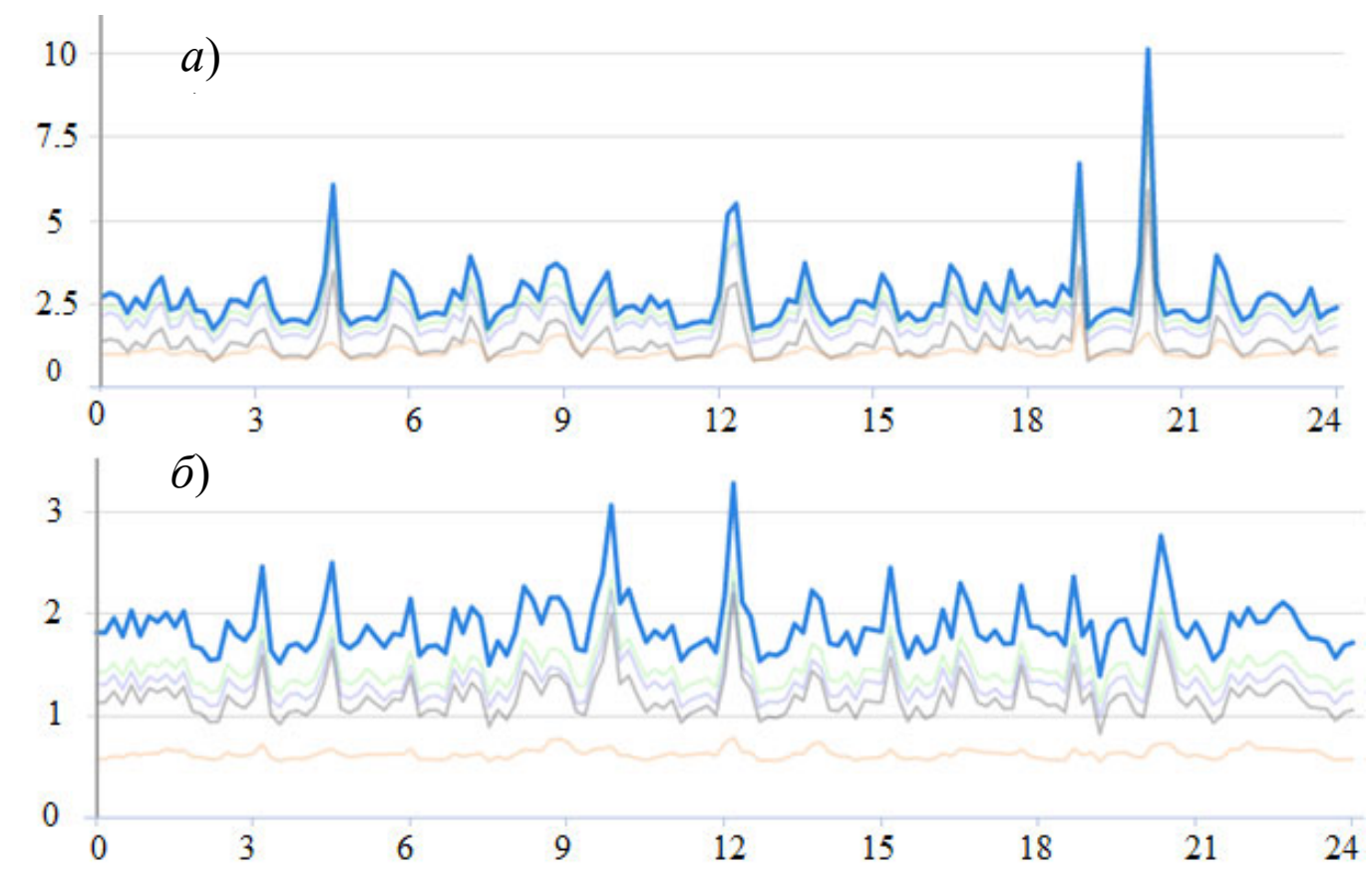

Рис. 3. Диаграмма GDOP на пункте BARE (02.01.2020):

а) ГЛОНАСС; б) ГЛОНАСC+GPS 
Кроме указанных выше расчетов, в программе Trimble Planning были выполнены вычисления геометрических факторов на широтах от $55^{\circ}$ до $75^{\circ}$, с шагом $5^{\circ}$. Для созвездий GPS и ГЛОНАСС на $70^{\circ}$ с.ш. наблюдается несколько кратковременных увеличений значения геометрического фактора до 4-6 единиц, a на $75^{\circ}$ с.ш. эта величина может достигать значения 8, что неожиданно для суммарной орбитальной группировки.

\section{Заключение}

Как показали расчеты, на северных территориях наблюдается достаточное количество спутников ГНСС для навигационного решения. На заполярном пункте BARE по всем азимутам спутники наблюдаются на высотах более $30^{\circ}$, в отличие от пункта NSK1 в средних широтах, где есть «окно» с низким расположением спутников в северном направлении.

На пункте Баренцбург величина геометрического фактора PDOP для суммарного созвездия спутников GPS и ГЛОНАСС не превышает значения 4 в течение суток в разные сезоны, что является хорошим показателем, при этом для созвездия исключительно спутников ГЛОНАСС в течение суток могут быть кратковременные увеличения геометрического фактора до $6-10$ единиц, в результате чего резко ухудшается качество решения. Этот факт следует учитывать при планировании ГНСС-измерений и выполнении навигации по спутникам ГЛОНАСС, при реализации метода точного точечного позиционирования в северных широтах, а также при обновлении или расширении орбитальной группировки ГЛОНАСС.

\section{БИБЛИОГРАФИЧЕСКИЙ СПИСОК}

1. Антонович К.М. Космическая навигация: учеб.пособие. - Новосибирск: СГУГиТ, 2015. $-233 \mathrm{c}$.

2. Войтенко А.В. О реализации и оценке точности методики «Precise Point Positioning (РРР)» // Геодезия и картография. - 2017. - Т. 78. - № 9. - С. 42-49.

3. ГКИНП (ОНТА)-02-262-02. Инструкция по развитию съемочного обоснования и съемке ситуации и рельефа с применением глобальных навигационных спутниковых систем ГЛОНАСС и GPS. - М: ЦНИИГАиК, 2002. - 124 с.

4. ГОСТ Р 52928-2008. Система спутниковая навигационная глобальная. Термины и определения. - М.: Стандартинформ, 2008. - $11 \mathrm{c}$.

5. ГОСТ Р 57372-2016 Глобальная навигационная спутниковая система. Методы и технологии выполнения геодезических работ. Пункты высокоточной геодезической сети (ВГС). Технические условия [Электронный ресурс]. - Доступ из справ.-правовой системы «КонсультантПлюс».

6. ГОСТ Р 57374-2016 Глобальная навигационная спутниковая система. Методы и технологии выполнения геодезических работ. Пункты фундаментальной астрономо-геодезической сети (ФАГС). Технические условия [Электронный ресурс]. - Доступ из справ.-правовой системы «КонсультантПлюс».

7. Липатников Л.А. О методике точного дифференциального позиционирования (Precise Point Positioning) и перспективах её совершенствования // Интерэкспо Гео-Сибирь. 2012. - T.7. - C. 48-53. 
8. Об установлении структуры государственной геодезической сети и требований к созданию государственной геодезической сети, включая требования к геодезическим пунктам [электронный ресурс]: Приказ Министерства экономического развития от 29.03.2017 г. №138. - Доступ из справ.-правовой системы «КонсультантПлюс».

9. Центр геодезии, картографии и инфраструктуры пространственных данных. РГСцентр [Электронный ресурс]. - Режим доступа: https://rgs-centre.ru/.

10. Центр геодезии, картографии и инфраструктуры пространственных данных. Список координат и скоростей пунктов, участвовавших в первичном построении системы координат ГСК-2011 на эпоху 1 января 2011 года [Электронный ресурс]. - Режим доступа: https://cgkipd.ru/opendata/fags/list.php?clear_cache $=$ Y.

11. Kaftan V., Malkin Z., Pasynok S., Pobedinsky G., Popadiev V. Reference Frames// National report for the International Association of Geodesy of the International Union of Geodesy and Geophysics 2015-2018. - Moscow, 2019. - P.8-26. DOI: 10.2205/2019IUGG-RU-IAG-8-26.

12. Leick A., Rapoport L., Tatarnikov D. GPS Satellite Surveying, 4th Edition. Published by John Wiley \& Sons Inc., 2015. - 840 p.

13. Li X. Precise positioning with current multi-constellation Global Navigation Satellite Systems: GPS, GLONASS, Galileo and BeiDou / X. Li, X. Zhang, X. Ren, M. Fritsche [at al.] // Scientific Reports. - 2015. - T. 5. - DOI: 10.1038/srep08328 [Electronic resource]. - Mode of access: https://www.ncbi.nlm.nih.gov/pmc/articles/PMC4321187/pdf/srep08328.pdf.

14. Trimble GNSS Planning Online [Electronic resource]. - Mode of access: https://www.gnssplanningonline.com/.

(C) Е. Г. Гиенко, А. С. Саливончик, 2021 